\title{
Über den Nutzen des Tuberkulins bei der Tuberkulose des Anges.
}

Ton

\author{
Prof. A. v. Hippel
}

in Göttingen.

Die höchst unbefriedigenden Resultate der Behandlung schwerer tuberkulöser Erkrankungen des Auges veranlassten mich vor melr als zehn Jahren zu therapeutischen Versuchen mit dem Tuberkulin. Die Bedenken, welche besonders von seiten der pathologischen Anatomen gegen die Anwendung des Mittels geltend gemacht wurden, erschienen mir für die Tuberkulose des Auges nicht massgebend, denn bei ihr lässt sich in den meisten Fällen eine gleichzeitige tuberkulöse Erkrankung innerer Organe nicht nachweisen, die Zahl der in den Geweben des Auges rorhandenen Bacillen ist erfahrungsgemäss eine sehr geringe, die Gefahr der Verschleppung derselben daher nicht gross. - Bei den ersten Patienten war ich noch auf den Gebrauch des alten Tuberkulins angewiesen; seit Bekanntgabe des neuen T. R. habe ich ausschliesslich dieses benutzt.

Über einige der von mir behandelten Fälle hat Schieck ${ }^{1}$ ) in seiner 1900 erschienenen Arbeit bereits berichtet. Dieselben reichen indessen nach meiner Ansicht nicht aus, um zu einem sichern Urteil über den Wert der Tuberkulintherapie zu gelangen. Ich habe daher meine Versuche fortgesetzt und befinde mich jetzt im Besitz genügender Erfahrungen, um das Mittel auf das Wärmste empfehlen zu können. Es rettet tatsächlich eine nicht geringe Zahl von Augen, die nach der heute geltenden, fast allgemeinen Anschauung der Enucleation verfallen sind, und zwar zum Teil mit Erhaltung eines brauchbaren Sehvermögens.

1) F. Schieck, Klinische und experimentelle Studien über die Wirkung des Tuberkulins auf die Iristuberkulose. v. Graefe's Areh. f. Ophthalm. Bd. L. Abt. 2.

v. Graefe's Archiv für Ophthalmologie. LIX. 1. 
A. v. Hippel.

Bei der Tuberkulose der Aderhaut habe ich bisher keine Versuche mit dem Tuberkulin angestellt, weil dieselbe in der Regel nicht unter einem so charalkteristischen ophthalmoskopischen Bilde auftritt, dass sie klinisch mit Sicherheit erkannt werden kann. Die Erkrankungen des vordern Augapfelabschnittes: des Corpus ciliare, der Iris, der Cornea, Sklera und der Conjunctiva sind es, bei denen das Tuberkulin besondern Nutzen bringt.

In allen Fällen von schwerer Iristuberkulose fand ich das Corpus ciliare und die Cornea mehr weniger in Mitleidenschaft gezogen, das klinische Krankheitsbild erscheint daher genügend charakterisiert, um zu einer sichern Diagnose zu gelangen, wenn auch die einzelnen Symptome graduelle Verschiedenheiten zeigen.

Neben lebhafter ciliarer Injektion findet sich eine den tiefen Schichten der Cornea angehörende, bald zentrale, bald mehr peripher gelegene Trübung, in welcher kleine und grössere umschriebene graugelbliche Herde, umgeben von einem lichteren Hof, sichtbar sind. Gegen dieselben ziehen aus den tiefern Randgefässen der Cornea stammende neugebildete Gefässe, welche nicht wie bei Keratitis parenchymatosa pinselförmig endigen, sondern mit den benachbarten anastomosieren und sich zwischen den einzelnen Herden netzförmig ausbreiten. An der Hinterfiäche der Cornea finden sich Präzipitate in verschiedener Zahl und Grösse, das Kammerwasser erscheint mehr weniger getrübt. Die gleichzeitig vorhandene Iritis tritt gewöhnlich in der Form der plastischen auf, ist mit starker Gefässneubildung in der Iris verbunden und führt schnell zu ringförmigen hintern Synechien und dicken Pupillarexsudaten. Bezeichnend für den tuberkulösen Charakter der Entzündung ist das Auftreten teils miliarer, teils konglobierter grosser Knoten von gelblicher Farbe im Ciliarteil der Iris. - Seltener erkrankt das Auge unter dem Bilde der sog. Iritis serosa, bei welcher die intensive Trübung des vordern Glaskörperabschnittes und die massenhaften Auflagerungen auf die Hinterfläche der Hornhaut eine frithe Beteiligung des Ciliarkörpers an der Entzündung beweisen.

Bei der Tuberkulose der Sklera entwickeln sich meist in der Nähe des Hornhautrandes bald einzeln stehende, bald miteinander konfluierende Knoten von verschiedener Grösse und gelblichgrauer Farbe, über denen die Conjunctiva verschieblich ist. Umgeben sind sie von einem Kranz erweiterter Gefässe. Durch das Fehlen tiefer venöser Injektion, eine schärfere Begrenzung und geringere Grösse unterscheiden sie sich von den bei Episkleritis auftretenden rundlichen Buckeln. 
Die Tuberkulose der Conjunctiva kann bekanntlich unter verschiedenen klinischen Bildern auftreten: bald in Form ron flachen oder tiefer greifenden Geschwüren, umgeben von mehr weniger zahlreichen miliaren Knötchen, welche die Tendenz zu frühzeitigem Zerfall zeigen; bald in Gestalt von graugelblichen, halbkugeligen Prominenzen, ähnlich den Trachomfollikeln; endlich als lebhaft gerötete papilläre Wucherungen, welche die Conjunctiva der Lider bedecken. In den meisten Fällen findet sich eine Mischung dieser Formen.

Überblickt man die Literatur, welche sich mit der therapeutischen Verwendung des Tuberkulins bei der Tuberkulose des Auges beschäftigt, so erscheint dieselbe ausserordentlich dürftig, denn abgesehen von einigen kurzen Mitteilungen aus dem Jahre 1891 unmittelbar nach der Bekanntgabe des Kochschen Mittels, welche keine sicher begründeten. Schlussfolgerungen gestatten (bezüglich der Literaturangaben verweise ich auf die Arbeit von Schieck), berichtet erst 1898 Zimmermann $^{1}$ ) über einen Fall, in dem das neue Tuberkulin T. R. zur Anwendung kam. Da in diesem aber die Tuberkel der Sklera und Cornea auch mit dem Galvanokauter behandelt wurden, so lässt sich nicht entscheiden, welchem Verfahren der schliesslich günstige Ausgang zu verdanken war.

Morax und Chaillous²) raten 1901 zur Anwendung des alten Tuberkulins bei Keratitis und Iritis, deren Ätiologie sich auf anderem Wege nicht sicher ermitteln lässt, und wollen in einem Falle nach einer einmaligen Injektion, die von hohem Fieber gefolgt war (die Dosis wird nicht angegeben), nach 14 Tagen Heilung erzielt haben(?).

Aus der Augenklinik in Tübingen berichtet Falckenberg ${ }^{3}$ ) 1901 über zwei Fälle, die mit Tuberkulin T. R. behandelt wurden. In dem ersten handelte es sich um ein dreijähriges Kind mit schwerster Hornhaut- und Iristuberkulose. Da neun Injektionen das weitere Wachstum der Tuberkelknoten nicht zum Stillstand brachten, wurde das Auge enucleiert. Patient starb fünf Monate später, Sektion unterblieb.

Der zweite betraf ein Kind von acht Jahren mit Kerato-Iritis und zahlreichen Knötchen in der Iris sowie beginnendem Skleral-

1) Die ophthalm. Klinik. 1898. S. 144 .

2) Annales d'oculist. 1901. Tom. CXXVII. p. $119 \mathrm{ff}$.

$\left.{ }^{3}\right)$ Ein Beitrag zur Pathologie und Therapie der Iridocyclitis tuberculosa. Dissertatio inaug. Tübingen 1901. 
A. r. Hippel.

staphylom. Zehn Tuberkulininjektionen hatten keinen sichtbaren Einfluss auf den Krankheitsprozess, daher Enucleation; vier Wochen später Tod durch Meningitis.

Im Jahre 1902 empfahlen Haab und Pflüger in der Sitzung der ophthalmologischen Gesellschaft in Heidelberg das Tuberkulin zu. diagnostischen Zwecken (Bericht S. 131), und v. Michel erwähnte, dass er gleichfalls in einem Fall von Iritis davon Gebrauch gemacht, in welchem nach wenigen Stunden zahlreiche Knötchen in der Iris und Sklera aufgetreten wären.

Handman ${ }^{1}$ ) berichtet in demselben Jahre über zwei Fälle von Kerato-Iritis mit Knötchenbildung, welche durch Injektionen mit Tuberkulin T. R. geheilt wurden.

Über drei Fälle von Conjunctivaltuberkulose, welche durch Tuberkulinbehandlung geheilt wurden, habe ich 1902 durch Salie ${ }^{9}$ eine kurze Mitteilung machen lassen. Da die Arbeit den meisten Fachgenossen unbekannt geblieben sein dürfte, werde ich später auf dieselbe nochmals zurückkommen.

1903 empfiehlt Enslin3) auf Grund seiner Beobachtungen in der Breslauer Augenklinik den Gebrauch des alten Tuberkulins bei ätiologisch unklaren Fällen von Keratitis parenchymatosa und betont als charakteristisch für die tuberkulöse Form das steile Ansteigen und schnelle Abfallen der Temperaturkurve nach der Injektion, eine Beobachtung, die mit meinen Erfahrungen vollkommen übereinstimmt.

Endlich erwähnt Haab ${ }^{\text {a }}$ nochmals die Verwendbarkeit des alten Tuberkulins zu diagnostischen Zwecken. Er hat in 21 Fällen davon Gebrauch gemacht und dabei nicht nur allgemeine, sondern auch lokale Reaktion am Auge beobachtet. Therapeutisch hat er sich des Mittels nur in einem Falle von Iritis mit Knötchenbildung und grossem Herde in der Chorioidea bedient, der durch 14 Injektionen geheilt wurde.

Weitere Mitteilungen uber den Gegenstand habe ich in der Literatur nicht gefunden. Die angeführten lehren, dass systematische

1) Handmann, Klin. Monatsbl. f.. Augenheilk. 1902. Bd. II. S. 219 ff.

9) Salie, Über die Erfolge der Tuberkulinbehandlung bei Conjunctivaltuberkulose. Inaug.-Dissert. Göttingen 1902.

3) Enslin, Über die diagnostische Verwertung des Alt-Tuberkulins bei der Keratitis parenchymatosa. Deutsche medicin. Wochenschrift. 1903. S. 130 u. 155.

4) Haab, Correspondenzblatt f. Schweizer Ärzte. 1903. Nr. 20. 21. 
Untersuchungen über den Einfluss des Tuberkulins auf die Tuberkulose des menschlichen Auges bisher fehlen. Diese Lücke auszufüllen, ist der Zweck der nachfolgenden Mitteilungen.

Bei Beginn meiner therapeutischen Studien hatte ich insofern mit Schwierigkeiten zu kämpfen, als mir jeder Anhalt für die zweckmässigste Dosierung des Tuberkulins, sowie für die Häufigkeit und Dauer seiner Anwendung fehlte. Vorsicht schien mir um so mehr geboten, als das alte Tuberkulin, welches in den ersten Fällen noch zur Verwendung kam, bekanntlich anfangs sehr ungleichmässig in seiner Wirkung war und nicht selten auch in kleinen Dosen heftige entzündliche Reaktionen an der Einstichstelle und starke Temperatursteigerungen hervorrief. Diese unliebsamen Zufälle, welche auch ich mehrfach beobachtete, veranlassten mich anfänglich wiederholt $\mathrm{zu}$ längerer Unterbrechung der Behandlung oder zum vorzeitigen Aussetzen der Tuberkulininjektionen, wodurch in eimigen Fällen Rezidive herbeigeführt wurden, die sich, wie spätere Erfahrungen lehrten, mit grosser Wahrscheinlichkeit bei konsequenter Anwendung des Mittels hätten vermeiden lassen. Seit der Benutzung des neuen Tuberkulins T. R. wurden diese unangenehmen Komplikationen selten und der Erfolg der Behandlung viel sicherer.

Nach mannigtachen Versuchen hat sich mir folgende Methode der Anwendung des Tuberkulins als die zweckmässigste erwiesen: ich beginne die Injektionen mit einer Dosis von $1 / 500 \mathrm{mg}$ der Trockensubstanz und wiederhole sie unter regelmässigen zweistïndigen Temperaturmessungen einen Tag um den andern, jedesmal tum ${ }^{1 / 500} \mathrm{mg}$ steigend. Eine tägliche Anwendung muss ich widerraten, weil bei manchen Patienten erst am Tage nach der Injektion eine Temperatursteigerung eintritt, die man zurückgehen lassen soll, ehe man von neuem injiziert. - Von $1 / 50 \mathrm{mg}$ ab erhöhe ich die Dosis bei jeder neuen Injektion um $1 / 50 \mathrm{mg}$, von ${ }^{10} / 50 \mathrm{mg}$ ab um ${ }^{4} / 50$ bis ${ }^{5} / 50$, sofern die Temperatur normal bleibt. Erhebt sie sich uiber $38^{\circ}$, so injiziere ich zwei- bis dreimal dieselbe Dosis und verstärke sie erst wieder, wenn der Patient keine allgemeine Reaktion mehr zeigt. Über $1 \mathrm{mg}$ der Trockensubstanz hinauszugehen, halte ich nach den Erfahrungen der letzten Jahre für überflüssig, denn es ist mir auch mit wesentlich kleinern Dosen gelungen, Fälle von schwerster Tuberkulose des Auges zu heilen. Warnen möchte ich davor, die Behandlung gleich mit grössern Dosen zu beginnen, weil ich in einigen Fällen von Tuberkulose der Cornea durch zu starke lokale Reaktion einen Zerfall des über dem Knoten liegenden Hornhautgewebes und dadurch 
bedingt die Entstehung eines Geschwüres beobachtete, das übrigens unter einem feuchtwarmen Verband bald zur Heilung gelangte.

Von grösster Wichtigkeit für die Erreichung einer dauernden Heilung ist es, das Tuberkulin lange genug anzuwenden. In den ersten Jahren habe ich wiederholt den Fehler gemacht, der Ungeduld der Kranken nachzugeben und mit den Injektionen aufzuhören, sobald die sichtbaren Tuberkelknoten in der Iris verschwunden waren, während Schwellung und Vaskularisation des Irisgewebes noch fortbestanden. Dies rächte sich regelmässig durch ein Rezidiv, das eine erneute Behandlung erforderlich machte. Die Injektionen müssen so lange fortgesetzt werden, bis alle Tuberkelknoten durch Narbengewebe ersetzt sind, die Schwellung und Vaskularisation der Iris sich zurückgebildet hat, Präzipitate an der Hinterfläche der Hornhaut und Glaskörpertrübungen verschwunden sind. $\mathrm{Um}$ in den schwersten Fällen definitive Heilungen zu erreichen, bedurfte es einer Behandlungsdauer von sechs Monaten und darüber.

Die Veränderungen, welche wir bei Bentzung der Zeissschen binokularen Lupe an tuberkulös exkrankten Augen unter der Einwirkung des Tuberkulins sich abspielen sehen, sind ja im einzelnen verschieden, zeigen aber im ganzen eine gewisse Regelmässigkeit in ihrem Verhalten.

Durch zahlreiche experimentelle Untersuchungen ist nachgewiesen, dass dasselbe nicht etwa die Bacillen tötet, sondern eine reaktive Entzitndung in dem diese umgebenden Gewebe hervorruft, in welchem es nach vorangegangener Exsudation zur Bildung von Narbengewebe kommt. Diesen $\nabla$ organg auch am erkrankten menschlichen Auge herbeizufuhhren, ist die Aufgabe der Behandlung. Sie wird nach meiner Erfahrung am besten gelöst durch die Anwendung Kleiner, ganz allmählich steigender Dosen von Tuberkulin, welche keine sichtbare Zunahme der am Auge wahrnehmbaren Entzündungserscheinungen hervorrufen sollen.

Bei diesem Verfahren pllegt nach zwei bis drei Wochen die Injektion des erkrankten Auges abzunehmen, die tief sitzenden Hornhauttrübungen beginnen etwas lichter zu werden, grosse Geschwulstknoten in der Iris erscheinen etwas kleiner und weniger vaskularisiert, von gleichzeitig vorhandenen miliaren Knötchen sind einzelne nicht mehr sichtbar. Im Laufe der nächsten Monate werden die Augen allmählich äusserlich vơllig reizlos, die Hornhauttrübungen hellen sich weiter langsam auf, die oft massenhaften Präzipitate an der Descemetis nehmen an Zahl und Grösse $a b$, die miliaren Knöt- 
chen in der Iris verschwinden spurlos, die grossen Knoten verkleinern sich fortgesetzt ohne zu zerfallen, bis schliesslich an ihrer Stelle sich ein graues, gefässloses Narbengewebe in der Iris bildet. Schwellung und Vaskularisation derselben verlieren sich erst nach Rückbildung aller Knoten; gefolgt sind sie in allen schweren Fällen von mehr minder ausgesprochener Atrophie des Trisgewebes. - Dicke, von Gefässen durchzogene Pupillarexsudate können sich im Laufe von Monaten zum grossen Teil resorbieren, eine Lösung der ausgedehnten Synechien gelingt dagegen meist nicht. Die in schweren Fällen stets vorhandenen Glaskörpertrübungen bilden sich unter der Einwirkung der Behandlung langsam, aber anhaltend zurück. Selbst ein dickes, gelbgraues Glaskörperexsudat in einem Falle schwerster tuberkulöser Iridocyclitis serosa habe ich in fünf Monaten völlig verschwinden sehen.

Abweichungen von diesem typischen Heilungsverlauf beobachtete ich nur in den Fällen, in welchen ich zu grosse Anfangsdosen von Tuberkulin angewandt oder die Dosis zu rasch gesteigert hatte. Unter Zunahme der Reizerscheinungen am Auge vermehrten sich dann entweder zunächst noch die Tuberkelknötchen in der Iris und Cornea, um erst nach Verkleinerung der Dosen allmählich zu verschwinden, oder es kam zu einem umschriebenen Zerfall des Gewebes in der Umgebung der Knötchen, so dass sich über denselben in der Hornhaut kleine Geschwüre bildeten. Bei rorsichtiger Anwendung des Tuberkulins lassen sich diese, die Heilung nicht hindernden, aber verzögernden Komplikationen vermeiden.

Rezidive habe ich wiederholt beobachtet, solange mir noch ausreichende Erfahrungen über die zweckmässigste Methode und die erforderliche Dauer der Behandlung fehlten. Seitdem ich dieselbe so lange fortsetze, bis alle entzündlichen Veränderungen in Cornea, Iris und Glaskörper völlig verschwunden sind, kamen Rückfälle bisher nicht mehr vor. Im ganzen habe ich im Laufe von zehn Jahren 23 Fälle von Tuberkulose der Iris, des Corpus ciliare und der Cornea, 1 ron Tuberkulose der Sklera, 3 von Tuberkulose der Conjunctiva (unter melnr als 50000 Patienten) behandelt. In den letzten wurde die Diagnose durch Impfung von Kaninchenaugen sicher gestellt. Bei der ersten Gruppe verbot sich dieses Experiment, weil ich die Ausführung der Iridektomie lediglich zu diagnostischen $Z$ wecken mit Rücksicht auf die Möglichkeit einer Verschleppung der Bacillen und eine durch das Trauma bedingte Steigerung der Entzündung für unzulässig halte. Von den erwähnten 23 Fällen scheide ich 13 von einer eingehenderen Besprechung aus, weil sie mir 
A. v, Hippel.

für den Nutzen des Tuberkulins nicht absolut beweiskräftig erscheinen. Bei ihnen handelte es sich neben mehr weniger schwerer Kerato-Iritis nur um die Entwicklung miliarer Knötchen in der Iris, die erfahrungsgemäss sich unter Umständen anch beim Gebrauch von Atropin und feuchtwarmen Verbänden spontan zurückbilden können, so dass ich mich nicht für berechtigt halte, die später eingetretene Heilung ausschliesslich dem Tuberkulin zuzuschreiben. Für unbedingt beweisend halte ich dagegen die übrigen zehn Fälle, in denen ausnahmslos schwerste Tuberkulose vorlag, welche jeder bisherigen Behandlung Trotz geboten hatte. Von diesen gebe ich nun zunächst Auszüge aus den sehr genan geführten Krankengeschichten, um jedem Leser ein eigenes Urteil über die Wirkung der Behandlung zu ermöglichen. Von einer Veröffentlichung der zahlreichen Temperaturkurven nehme ich Abstand; jede erheblichere Temperatursteigerung ist in den Krankengeschichten erwähnt. Ư ber die Fälle 1, 3, 4, 5 hat Schieck ${ }^{y}$ bereits berichtet. Ich nehme sie nochmals hier auf, um einen zusammenfassenden Überblick über alle in Frage kommenden zu geben.

1. Emma H., 25 Jahre, Alterode. I. Aufnahme in die Klinik 30. X. 1892. Keine hereditäre Belastung, keine Lnes, Lungen sonst normal, nur leichte Dämpfung über der rechten Spitze, wenig Auswurf, im Sputum keine Bacillen. Rechtes Auge normal, $\mathrm{M}=0,75 \mathrm{D}, \mathrm{S}=1$; auf dem linken Auge seit drei Wochen Abnahme des Sehvermögens.

Status: Geringe ciliare Injektion, einzelne feine Beschläge an der Descemetis, Iris etwas hyperämisch, Pupille auf Atropin maximal erweitert. Glaskörper diffus rauchig, einige bewegliche Flocken, Fundus leicht verschleiert, normal. E. $\mathrm{S}<1$. - Eine melrrwöchentliche Schwitzkur bewirkte keine Bessertung.

21. XI. Kleines Knötchen unten aussen im Kammerfalz, Iris grünlich verfärbt. Glaskörper viel stärker getrübt, S Finger in $2 \frac{1}{2}$ Meter. - In den nächsten Wochen allmähliches Wachstum des Knötchens gegen die Mitte der Iris unter zunehmender Vaskularisation.

17. XII. Ein zweiter Knoten bildet sich innen unten, bis zum 30.XII. sind beide miteinander konfluiert und umgreifen fast die nntere Irisperipherie.

Drei Injektionen mit altem Tuberkulin von $0,1-0,2-0,5 \mathrm{mg}$ am 9.-11.-14. I. 1893 riefen weder allgemeine noch lokale Reaktionen hervor, wurden daher nicht fortgesetzt.

Unter Atropin und Kataplasmen gingen die Reizerscheinungen in den nächsten Woohen zurück, die Knoten verkleinerten sich und versehwanden schliesslich mit Hinterlassung einer grauen Leiste unten im Ciliarteil der Iris. Die Glaskörpertrübung lichtete sich nicht. Bei der auf' Wunsch erfolgten Entlassung am 24. II. 1893 ersehien das Auge reizlos, die

1) Loc. cit. 
Pupille prompt reagierend, am hintern Linsenpol eine leichte Tribung. $\mathrm{S}$ Finger in $3 \mathrm{~m}$. Rechtes Auge normal, $\mathrm{S}=1$.

II. Aufnahme 6. V. 1893. Seit drei Wochen sollen auf beiden Augen Entzündungserscheinungen aufgetreten sein.

Status: Rechts ciliare Injektion, Cornea und Humor aqueus klar; im unteren äusseren Quadranten der Iris im Ciliarteil ein apfelkerngrosser Knoten von graugelblicher Farbe, stark vaskularisiert, bis zur Mitte der Iris reichend. Drei hintere Synechien, Glaskörper diffus getrübt, Fundus verschleiert, aber normal. M $1,5, \mathrm{~S}<0,8$.

Links: Ciliare Injektion, einige Präzipitate an der hintern Hornhautwand; im untern Kammerfalz ein grangelblicher flacher vaskularisierter Knoten, der aussen bis zum horizontalen Meridian heraufreicht und hier an zwei hirsekorngrosse Knötchen von gleicher Beschaffenheit stösst, die auch im Kammerfalz liegen. Glaskörpertrübungen dichter, Hintergrund nicht sichtbar, Finger in $2 \mathrm{~m}$. - Patientin wurde mit Atropin und Kataplasmen behandelt und am 3. VII. anf ihren Wunsch entlassen, ohne dass eine Besserung eingetreten war.

20. VII. 1893. Vorstellung. Linkes Auge: Knoten gewachsen, völlig miteinander konfluiert, umfassen mehr als die untere Hälfte der Iris. An der medialen Seite noch ein neuer Knoten, der aus dem Kammerwinkel in die Iris hinein gewachsen ist. Alle Knoten berühren fast die hintere Hornhautwand; an letzter zahlreiche Präzipitate.

Rechts: Stat. idem.

III. Aufnahme 18. IX. 1893. Rechtes Auge: Unten aussen im Ciliarteil der Iris ein $5 \mathrm{~mm}$ langer, graurötlicher Knoten mit stark vaskularisierter höckeriger Oberfläche, der fast die Cornea berïhrt und sich gegen den Pupillarrand abflacht. Pupille durch Atropin erweitert, Linse dnrchsichtig, diffuse Trubung des Glaskörpers, Hintergrund nicht sichtbar, $\mathrm{s}<0,3$.

Linkes Auge: Präzipitate zahlreicher. Die untern Zweidrittel der Irisperipherie sind von einem gelbgrauen Tumor eingenommen mit höckeriger, stark vaskularisierter Obertläche. Pupille fast total adhärent, zum Teil von dünnem Exsudat erfuillt. Fundus nicht sichtbar. $S=$ Handbewegungen, Lichtschein und Projektion normal.

25. IX. Infolge Zunahme der Glaskörpertrübungen ist auf dem rechten Auge $S$ auf 0,1 gesunken, daher am 29. IX. 1893 Beginn mit konsequenter Tuberkulinbehandlung. Erste Dosis 0,05 mg altes Tuberkulin, anfangs jeden zweiten, später jeden dritten Tag um $0,05 \mathrm{mg}$ steigend. Am 25. I. 1894 Injektionsdosis $5,0 \mathrm{mg}$; von da ab wurde erst um $1 \mathrm{mg}$, dann um $2 \mathrm{mg}$ bis zur Enddosis von $18 \mathrm{mg}$ am 1. III. 1894 gestiegen. Injektionen gut vertragen, keine wesentlichen Temperatursteigerungen bis auf eine nach Injektion von $4 \mathrm{mg}$, nach welcher $38,6^{\circ}$ and Mattigkeitsgefühl eintraten. Im ganzen kamen 45 Injektionen zur Verwendung.

Bereits am 17. X. deutliche Verkleinerung aller Tumoren. Bis zum 23. XI. hatten sie sich in gran gefärbte atrophische Leisten umgewandelt, die Injektion der Augen war versehwunden.

Am 4. XII. erschien das linke Auge schon so reizlos, dass ich mit Rücksicht auf die totale hintere Synechie eine Iridektomie nach oben aus- 
führte. Dieser Eingriff erwies sich als verfüht, denn es $\mathrm{kam}$ zu einer Exsudation in das Pupillar- und Colobomgebiet, das Auge injizierte sich and am 13. I. 1894 erschien die Leiste auf der Iris etwas geschwellt und vaskularisiert, während das rechte Ange reizlos blieb und die Rückbiläung der Tumoren fortschritt. Unter weiteren Injektionen ging der Anfall bald zuritiek.

Bei der Entlassung am 13. IIT. 1894 wurde folgender Befund erhoben: Rechtes Auge reizlos, Cornea und Humor aqueus klar, Iris sonst normal, nur unten aussen an Stelle des Tumors eine bindegewebige, von atrophischer Iris umgebene Leiste. Linse klar, Glaskörper wesentlich aufgehellt, Fundus verschleiert sichtbar, normal. $S=0,3$.

Linkes Auge: Geringe ciliare Injektion, Präzipitate weniger zahlreich, Cornea und Kammerwasser klar. An Stelle der Tuberkelknoten eine graue atrophische Leiste, auf deren Oberfläche noch einige Gefässe sichtbar sind. In Colobom und Pupille ein in Organisation befindliches Exsudat, kein rotes Licht aus dem Fundus, Finger $1 \mathrm{~m}$.

12. IV. 1894. Vorstellung. Rechtes Auge wieder injiziert, Iris atrophisch, die graue Leiste unverändert, aber unten im Kammerfalz wieder zwei kleine Knötchen. Glaskörper traber, $s=0,1$.

Linkes A uge reizlos, Iris überall atrophisch, Exsudat in Pupille und Colobom gesehrumpft. Projeltion nomal.

23. V. 1894. IV. Aufnahme. Linkes Auge reialos geblieben.

Am rechten Auge sind die Knötchen gewachsen und konfluiert, prominieren in die vordere Kammer bis nahe an die Hornhant und nehmen ungefähr Zweidrittel der Irisbreite ein. Präzipitate zahlreicher, Glaskôrper dicht getuibt, Hintergrund nicht siehtbar, Finger in $3 \mathrm{~m}$.

Vom 26. V. bis 19. VII, 189417 Injektionen mit Tuberkulin T. R. in zwei- bis viertagigen Intervallen, mit $0,5 \mathrm{mg}$ beginnend, anfangs um $0,1 \mathrm{mg}$, dann von $1 \mathrm{mg}$ ab um $0,2 \mathrm{mg}$, von $2 \mathrm{mg}$ ab um $0,5 \mathrm{mg}$ steigend bis zur Enddosis von $5 \mathrm{mg}$. Drei Wochen nach Beginn der Injektionen Auge reizlos, Knoten in deutlicher Rückbildung, die bis zum 23. VII. vollendet war. Cornea klar bis anf eine kleine Stelle ibber dem äusseren Pupillarand in den mittleren Parenchymlagen, zu der einige feine Gefässe verlaufen. Iris hochgradig atrophisch, Linse getrübt, ihre Kapsel verdickt, Glaskörper stark getrubt, Projektion normal. Linkes Auge reizlos, Atrophie der Iris starker, Projektion normal. Von da ab blieben die Augen von einem Rezidiv der Tuberkulose verschont.

16. III. 1895. Extraktion der Linse links, danach Pupillarverschluss durch schleichende Oyclitis.

23. VI. 1895. Iridotomie mit Seherenpincette, klare zentrale Pupille, aber infolge der Glaskörpertribungen schlehtes Selvermögen: Finger in $1 / 4 \mathrm{~m}$.

14. V. 1896. Iridektomie rechts mit normalem Verlauf; Glaskörper gleichfalls stark getribut. Finger in $6 \mathrm{~m}$.

10. X. 1900. Letrite Vorstellung, Status idem.

24. I. 1904. Laut brieflicher Mittelung ist kein Rückfall melr eingetreten. 
Epikrise: Die 25jährige Patientin erkrankte Anfang Oktober 1892 unter den Erscheinungen einer Iritis serosa des linken Auges. Ende November bildete sich ein Knötchen aussen unten im Kammerfalz, ein zweites entstand ungefähr drei Wochen später. Beide wuchsen, konfluierten und hatten sich bis Ende Dezember 1892 über einen Teil der untern Irishälfte ausgebreitet. Unter Atropin und Kataplasmen bildeten sie sich bis zum Februar 1893 zurïck mit Hinterlassung einer atrophischen Stelle in der Iris, die gleichzeitig vorhandenen Glaskörpertrübungen blieben aber bestehen. Im Mai 1893 Rezidiv auf beiden Augen, das unaufhaltsam fortschritt, so dass beiderseits die Iris zum grossen Teil von konfluierten Tuberkelknoten eingenommen war. 45 Injektionen mit altem Tuberkulin vom 29. IX. 1893 bis 13. III. 1891 in der Dosis von 0,05 bis $18,0 \mathrm{mg}$ führten eine völlige Rückbildung derselben herbei, aber zwei Monate später kam es nochmals zu einem Rückfall auf dem rechten Auge, und dauernde Heilung wurde erst erreicht durch 17 Injektionen mit dem neuen Tuberkulin T. R. Trotz der ausserordentlichen Schwere der Erkrankung gelang die Erhaltung eines zur Orientierung ausreichenden Sehvermögens.

2. Minna Bl., 24 Jahre, Gerberfrau, Mansfeld, stellte sich zuerst am 15.I. 1894 mit ehroniseher Iritis und Pupillarverschluss des linken A uges vor. Iridektomie. Im Colobom bleibt ein Teil der Pigmentschicht auf der Kapsel haften. Mit reizlosem Auge 7. III. 1894 entlassen. Finger in $1^{1 / 2} \mathrm{~m}$. Rechts E. $\mathrm{S}=1$.

Seit Februar 1896 Abnahme des Sehvermögens auf dem rechten Auge.

5. V. 1896 Aufnahme in die Klinik. Rechtes Auge: Zahlreiche Präzipitate an der Hornhaut, in der Iris vier grosse und eine Anzall miliarer Knoten von gelbgrauer Farbe und starker Vaskularisation; breite Synechien, Hintergrund infolge diffuser Glaskörpertrübung undeutlich, $S=0,5$.

Linkes Auge: Pupille und Colobom durch ein dickes Exsudat geschlossen, ganze untere Hälfte der Iris von einer grangelblichen vaskularisierten Geschwulst eingenommen, Cornea davor rauchig getrübt, viele Präzipitate. Lungen und Herz gesund (medizinische Klinik).

Vom 6. V. bis 12. VII. 1896 . 26 Injektionen ron $1 / 500$ bis $5 \mathrm{mg}$ T. R., während deren rechts sich alle Tuberkelknoten zurückbilden und bis auf eine Anzahl Präzipitate die Medien klarer werden, so dass $\mathbf{S}$ auf 0,8 steigt.

Am linken Auge ist der grosse Knoten in der untern Irishälfte ganz geschwunden, ebenso die kleinen, doch erscheint das Irisgewebe im Gegensatz zu rechts noch aufgelockert und vaskularisiert. Pupillarexsudat nnverändert.

15. VII. 1896. Auf Wunsch der Patientin Entlassung.

23. XI. 1900. Erst $4 \frac{1}{2}$ Jahre später stellte sie sich wieder vor, ob- 
gleich das Selvermögen allmählich abgenommen und das linke Auge sich öfter entzündet hatte.

Status. Rechtes Auge: Mässige ciliare Injektion, Cornea im Zentrum leicht parenchymatös getrübt, einzelne Präzipitate, Kammer flach, Iris verfärbt, von weiten Gefässen durchzogen, Synech. post. tot, dickes vaskularisiertes Pupillarexsudat. Oben aussen im Kammerfalz ein gegen die Cornea vorspringender grosser Knoten; Bulbus weich, Projektion fehlt.

Linkes Auge: Lebhafte Injektion, Cornea von Gefässen durchsetzt, diffus getrubt, parazentral eine Perforationsöffinung, aus der ein gelblich weisser Pfropf, von Gefässen bedeckt, heraustritt. Dieser hängt mit einem fast die ganze Kammer einnehmenden käsigen Exsudat zusammen, von zerfallenen Tuberkeln herrührend. Irisperipherie sichtbar, in ihr einige miliare und ein grösserer Knoten. Lichtschein vorhanden, Projektion falsch.

33 Injektionen von $1 / 600$ bis $1 / 2 \mathrm{mg}$ T. R. rom 24. XI. 1900 bis 3. II. 1901 bewirkten zwar die allmähliche Rückbildung der Knoten und eine Resorption des Exsudats auf dem linken Auge, konnten aber die fortschreitende Phthisis nicht mehx hindern, so dass Patientin am 8. II. 1901 erblindet entlassen werden musste.

Epikrise: Patientin, seit Juni 1892 an linksseitiger Iritis mit Pupillarverschluss leidend, wird erst 1894 iridektomiert und mit reizm losem Auge entlassen. Zwei Jahre später kommt sie mit doppelseitiger typischer Iristuberkulose zur Aufnahme. Die Tuberkelknoten werden durch $26 \mathrm{~T}$. R.-Injektionen von $1 / 500$ bis $1 / 2 \mathrm{mg}$ in zwei $\mathrm{Mo}-$ naten zur Rückbildung gebracht, aber das Irisgewebe des linken Auges ist bei der auf dringenden Wunsch der Patientin erfolgenden Entlassung noch geschwollen und vaskularisiert. Erst $4 . \%_{2}$ Jahre später stellt sie sich mit schwerstem Rezidiv auf beiden Augen und Perforation der linken Cornea wieder vor. 33 weitere Injektionen bewirken zwar Rückbildung der Tuberkelknoten, können aber den Fortschritt der Phthisis nicht hindern. - Auch in diesem Fall ist die Behandlung zum erstenmal nicht lange genug fortgesetzt worden.

3. Berthold B., 13 Jahre alt, Gutenberg, kam am 13. I. 1895 wegen beiderseitiger, seit 14 Tagen bestehender Augenentzïndung in die Klinik. Er will früher nie an den Augen gelitten haben, im 7. Jahre hatte er Schwellungen der Halsdrüsen und eine linksseitige eitrige Ohrenentzündung. Beide Eltern leben and sollen gesund sein.

Status: Schwächlicher Knabe. Keine Drüsenschwellungen, keine Zeichen von Lues oder Tuberkulose.

Beiderseits starke conjunctivale und ciliare Injektion. Cornealepithel matt, in den vordern Schichten einzelne kleine Trübungen, rechts ansserdem eine ungefähr $1 \mathrm{~mm}$ im Durchmesser haltende Trübung in den tiefern Sclichten parazentral unten. Vordere Kammer vertieft, Hum. aqueus leicht getrübt. Iris hyperämisch, geschwellt, Pupille durch Atropin mittelweit, 
Glaskörper diffus getrübt, Hintergrund verschleiert. S wegen Lichtscheu nicht $z u$ bestimmen.

In den nächsten Tagen trotz Atropin und Kataplasmen Zunahme der Entzündung unter Bildung mehrerer Synechien, zugleich Anschwellung des linken Kniegelenkes mit Druckempfindlichkeit des Condyl. int. tibiae und leichtem Fieber $(37,7)$ bei stark beschleunigtem Puls (124) und elendem Aussehen.

24. I. Am linken Auge făllt eine Vorwölbung der Sklera in der innern Hälfte des Limbus auf, die in den nächsten Tagen stärker wird, sich auf die Gegend des Corp. ciliare erstreckt, fast den ganzen Cornealrand umgreift and sich auch am rechten Auge bemerkbar macht. Kniegelenk nach wie vor geschwollen, bei Bewegungen sehr sehmerzhaft. Temperatur 37,7 , Puls 100 , phthisischer Habitus, aber in den Lungen nichts nachzuweisen (med. Kinik).

28. I. Auf der Iris des linken Auges heute oben innen ein vaskularisiertes Knötchen, subjektive Reizsymptome sehr stark. Cornea trübt sich immer mehr unter gleichzeitiger Getässnenbildung.

31. I. Ein zweites Knötchen zeigt sich oben aussen in der Iris. Pupille trotz Atropin eng.

Sehwellung des Knies geht zurück.

1. II. Heute auch in der Iris des rechten Auges zwei Knötchen, Ektasie der Sklera beiderseits unverändert, Hornhäute noch mehr getrübt, Iris sohr hyperämisch.

6. II. Die Knötchen in der Iris nehmen an Zahl $\mathrm{zu}$, Cornea in den tiefsten Schichten vaskularisiert, im Zentrum einige rundliehe gelbgraue Trübungen.

In den nächsten Tagen beginnen die Knötehen zu konfluieren und so schnell zu wachsen, dass sie fast die Hornhaut berühren, während die skleralen Buckel sich gleichfalls vergrössern. - Mit Rücksicht auf das rapide Wachstum der Tuberkelknoten und die Schwere der Entzündung wurden am 6. II. die T. R.-Injektionen gleich mit der Dosis von $1 / 20 \mathrm{mg}$ begonnen, Tag um Tag um $1 / 20 \mathrm{mg}$, von $1 / 2 \mathrm{mg}$ ab um $1 / 10 \mathrm{mg}$ erhöht. Von $1 \mathrm{mg}$ ab erfolgten sie in zweitägigen Intervallen unter Steigerung jeder Dosis um $2 / 10 \mathrm{mg}$ bis zur Höhe der Enddosis von $1,6 \mathrm{mg}$, welche am 15. III. erreicht wurde. Ihre Zahl betrug im ganzen 18 .

In den ersten Wochen der Behandlung nahm die Vaskularisation der Cornea, die parenchymatöse Infiltration der Iris und das Wachstum der Knoten noch zu. Ende Februar trat beiderseits ein Hypopyon auf und es kam zu Schwellung der präaurikularen und submaxillaren Drüsen.

Gegen die Mitte des März macht sich eine geringe Aufhellung der Hornhänte bemerkbar, die allmählich fortsehreitet, so dass man ein dickes, gelbliches Exsudat zentral an ihrer Innenfläche erkennt. Dieses verkleinert sich bis Ende März soweit, dass man beiderseits wieder die Knötehen in der Iris sehen kann.

In der ersten Halfte des April nehmen die äussern Entzündungserscheinungen $\mathrm{ab}$, die Aufhellung der Hornhäute schreitet fort, die Knötchen in der Iris verschwinden, die skleralen Prominenzen bilden sich zurück, eine leichte Verdünnung der Sklera hinterlassend. Bis Mitte Mai 
hatten sich die tiefen gelbgrauen Hornhautinfltrate in weissliche Trubungen verwandelt, von den Knötchen im Irisgewebe war keine Spur mehr sichtbar. An der Hinterfläche der Cornea noeh zahlreiche Präzipitate.

31. V. 1895. Entlassung. Augen reizlos, Hornhäute noch ziemlich stark getribt, Gefässe in der Rukkbildung. Vordere Kammer flach, Iris atrophisch, Pupillen von schrumpfendem Exsudat eingenommen, Hintergrund nicht sichtbar, Lichtschein and Projektion normal.

16. X. 1895. Vorstellung. Status sonst unverändert, nur in der atrophischen Iris beiderseits wieder einige kleine, reizlos im Gewebe eingebettete Knötchen.

13. II, 1896. Patient hat inzwischen eine Pleuropneumonie durchgemacht. Augen reizlos, die kleinen Knötchen spontan versehwunden.

6. V. 1896. Hornhäute noch weiter aufgehell, daher beiderseits Iridektomie; rechts: Finger vor dem Auge, links in $3 \mathrm{~m}$.

15. V. 1897. Augen reizlos; rechts Finger in $1 \mathrm{~m}$, links in $3 \mathrm{~m}$. Hornhanttribungen noch recht erheblich.

Epikrise: Bei einem an schwerer Tuberkulose der Iris, Cornea und Sklera erkrankten Knaben won 13 Jahren, bei dem sich die Entzündung der Augen im Laufe eines Monats zu solcher Höhe steigerte, dass beiderseits das Sehvermögen aufgehoben war, wurde der Versuch gemacht, durch T. R.-Injektionen in erheblich grösserer Anfangsdosis und schnellerer Steigerung der" weitern Ausbreitung des Prozesses entgegenzuwirken. Obgleich während der ganzen Dauer der Behandlung die Temperatur niemals über 37,8 stieg, riefen die Injektionen zunächst eine offenbar $z$ starke Reaktion an den Augen hervor, die sich durch Zunahme der Trübung und Vaskularisation der Cornea und teilweisen Zerfall der Tuberkelknoten in der Iris und Auftreten ron Hypopyon äusserte. Ihr giunstiger Einfluss kam aber trotzdem nachträglich zur Geltung und führte trotz des jugendlichen Alters des Patienten, welches die Prognose ungünstig beeinflusste, schliesslich Heilung herbei, die zwei Jahre hindurch konstatiert werden konnte.

4. Minna H., 14 Jahre alt, Wernigerode.

25. IX. 1896 Aufnahme in die Klinik. Eine Schwester der Mutter ist an Darmtnberkulose gestorben, ein Bruder der Patientin leidet an Lupus. Sie selbst war stets schwächlich und bat vor fünf Jahren an einer Erkrankung der Wirbelsäule gelitten, so dase sie wochenlang im Streekbett liegen musste. Die Eltern sind gesund. Seit ${ }^{1 / 4}$ Jahr besteht eine Entründung des linken Auges, die bisher erfolglos behandelt wurde.

Status: Rechtes Auge gesund, E. $\mathrm{S}=1$.

Linkes Auge: Starke conjunctivale und ciliare Injektion. In der oberen Hornhauthallte mehrere tief sitzende Infiltrate, intensiv weiss gefârbt, von einem lichten Hof umgeben, zu denen vom Limbus her tiefliegende, sich verästelnde Gefässe ziehen. Iris verfärbt, byperämisch, Synechia post. 
totalis; auf der vorderen Kapsel einzelne grauweisse Auflagerungen. Hintergrund rötlich, Einzelheiten nicht sichtbar.

Da jede sonstige Therapie erfolglos blieb, die Infiltrate an Zahl noch zunahmen und am obern Limbus drei subconjunctival gelegene, geibrötliche kleine Knoten auftraten, wurde im Hinblick auf die hereditäre Belastung am 17. X. 1896 mit T. R.-Injektionen begonnen, die bis zum 27. XI. fortgesetzt wurden. Im ganzen kamen 20 Injektionen von $1 / 20-1,4 \mathrm{mg}$ zur Anwendung.

Während der Behandlung entstand aus einem parazentralen Infiltrat ein Uleus mit weisslich infiltriertem Grund und die subconjunctivalen Knoten am obern Limbus vergrösserten sich, während am innern sieben neue auftraten. Am 27. XI. wurden daher die Injektionen ausgesetzt.

Anfang Dezember traten noch neue kleine Knoten am äussern Limbus auf, gegen Ende Dezember ging aber die Entzündung zurïck, die Infiltrate verwandelten sich in grauliche Trübungen, die Knoten am Limbus schwanden allmählich unter Hinterlassung einer leichten Verdünnung der Sklera.

Am 10. II. 1897 wurde Patientin geheilt entlassen. S Finger in $1 \mathrm{~m}$. 15. IV. 1897. Wegen Seclusio schmale Iridektomie nach innen unten. Das Pigmentblatt der Iris bleibt im Bereich des Coloboms teilweise auf der Kapsel haften; normale Heilung.

Nach einer brieflichen Mitteilung vom 23. I, 1904 ist das Auge von jeder Entzündung frei geblieben.

Epikrise: Auch in diesem. Fall wurde durch zu hohe Anfangsdosen und $\mathrm{zu}$ schnelle Steigerung derselben eine zu starke lokale Reaktion hervorgerufen, obgleich die Temperatur niemals über 37,5 stieg. Ein dauernder Schaden erwuchs der Patientin indessen daraus nicht, sondern die Tuberkulinbehandlung führte trotz der Schwere der Erkrankung eine dauernde Heilung herbei.

5. Marie Kr., 28 Jahre alt, Bergmannsfrau, Badersleben.

22. XI. 1898 Aufnahme in die Klinik. Patientin hat im siebenten Jahre eine skrofulöse Keratitis auf dem linken Auge gehabt und sieht seitdem schlecht damit. Das rechte war gesund, bis es sich vor fünf Monaten auch entzündete. Familienanamnese belanglos. Lues geleugnet, objektive Zeichen dafür fehlen. Über der linken Lungenspitze unreines Atmen; verlängertes, etwas hauchendes Exspirium (medizinische Klinik).

Status: Linkes Auge reizlos, zentrale Maculae corneae, sonst normal. $\mathrm{S}=0,3$.

Rechtes Auge: Starke Lichtschen, Tränen, lebhafte ciliare Injektion, Cornea rauchig getrübt, Epithel gestichelt, im äussern obern Quadranten ein grosses gelbgraues, tiefsitzendes Infiltrat, zu dem eine Anzahl tiefliegender Gefässe zieht. Vom innern Rande verlaufen zahlreiche Gefässe in den hintersíen Schichten zu einer, vor der Descemetis gelegenen dreieckigen gelbgranen Trübung, hinter welcher im Kammerfalz eine Anzahl von der 
Iris ausgehender, konfuierter Knoten liegt. Iris hyperämisch, einige Synechien. Vom 2. XII. 1898 bis 8. II. 189924 T. R.-Injektionen mit 1/500 $\mathrm{mg}$ beginnend und bis $1 \mathrm{mg}$ steigend.

In den ersten 14. Tagen der Behandlung traten noch drei neue graugelbliche Knötchen in den tiefsten Schichten der Cornea auf; die Injektion und die heftigen subjektiven Reizerscheinungen gingen aber im Laufe von drei Wochen völlig zuriek.

Nach vier Wochen begann eine Rüekbildung der Knoten in der Comea und im Kammerwinkel. Statt der ersten bildeten sich weissliche Tribungen, die sich allmählich immer mehr lichteten, so dass man nun auch eine Anzahl von ziemlich dichten Präzipitaten an der Hornhaut erkennen konnte, die hinter jenen lagen.

Am 9. II. 1899 wurde Patientin geheilt entlassen, $S=0,5$.

Status: Ange reizlos, vom innem Limbus erstreckt sich eine zungenförmige Trübung in die tiefern Lagen der Cornea. Sie ist so transparent, dass man die Iris dahinter sieht, die noeh einzelne erweiterte Gefässe zeigt, aber keine Knoten mebr.

6. III. 1899, Hornhauttrübungen bedeutend lichter, Ange reizlos, 5 rechts $=0,6$.

9. VI. 1899. Weitere Aufhellung. $\mathrm{S}$ rechts $=0,8$.

Am 7. VI. 1904 stellte sich Patientin in Göttingen mir nochmals vor. Das rechte Auge war gesund geblieben. $S=0,8$. Seit drei Wochen hatte sich aber in der Cornea des linken oben-aussen unter mässiger Injektion ein hirsekorngrosser, graugelber Knoten gebildet. An der Iris bestehen keine pathologischen Veränderungen (Zusatz bei der Korrektur).

Epikrise: Eine schwere Kerato-Iritis mit Knotenbildung in Cornea und Iris wurde durch 24 T. R.-Injektionen in Zeit von zwei Monaten geheil, obgleich in den ersten drei Wochen die Zahl der Knötchen noch zunahm. Beobachtungsdauer: fünf Jahre.

6. Hermann A., 20 Jahre alt, Zerbst.

3. XII. 1899. Patient hat stets gut gesehen, bis sich das linke Auge vor $21 / 2$ Wochen entzindete. Keine Lies, Lungen gesund (medizinische Klinik).

Status: Rechtes Auge normal, $\mathrm{E}, \mathrm{S}=\mathbf{1}$.

Linkes Ange: Jebhafte ciliare Injektion, Cornea leicht parenchymatös getrübt, Epithel matt, Vorderkammer vertieft, Humor aqueus getrubt, kleines Hyphäma, Pupille anf Atropin unregelmässig, fast total adhärent, Irisgewebe stark geschwollen, im untern äussem Quadranten ein gelblicher vaskularisierter Knoten, dickes Pupillarexsudat. - Unter Atropin, Kataplasmen, Verband steigerte sich die Eutzündung in Laufe der nächsten Woche und es bildeten sich oben in der Iris noch neve Knoten.

19. XII. Cornea matt, im Zentrum eine tief liegende Tribung, zu der zahlreiche, im Parenchym verlaufende Gefässe ziehen, Vorderkammer hoehgradig abgeflacht. Iris stark vorgetrieben, in ihrem obern Umfange vier grosse, zum Teil konfluierende, vaskularisierte gelbliche Knoten. Ein weiterer grosser Knoten liegt anssen im Ciliarteil, nuten finden sich noch 
drei etwas kleinere. Innen oben ist die Iris frei von Knoten, aber verdickt und von weiten Gefässen durchzogen. Pupille etwas erweitert, aber durch ein dickes Exsudat völlig verschlossen, das im Zentrum bis an die Hinterfläche der Hornhaut reicht.

Vom 21. XII. 1899 bis 8. II. 190023 T. R.-Injektionen von 1/500 bis $1 \mathrm{mg}$. Keine fieberhafte Reaktion. Nach drei Wochen begann die Rückbildung der Knoten und die Entzündungserscheinungen nahmen $a b$, jedoch blieb die totale Synechie bestehen und die innere Hälfte der Iris wölbte sich so weit vor, dass sie fast die Hinterfäche der Cornea berührte. Bis zum 8. II. 1900 waren alle Knoten versehwunden unter Hinterlassung einer ausgesprochenen Atrophie des Irisgewebes, und das Auge erschien reizlos. Wegen des Pupillarverschlusses wurde am 5. III. eine Iridektomie ausgefuhrt and Patient am 14. III. 1900 geheilt entlassen. S Finger in $2 \mathrm{~m}$.

4. IV. 1900. Letzte Vorstellung. Ange reizlos, Iris zum grossen Teil atrophisch, keine Knoten mehr sichtbar.

Am 26. I. 1904 berichtet Patient brieflich, dass das Auge sich nicht wieder entzïndet habe.

Epikrise: Eine schwere einseitige Iritis tuberculosa, welche mit der Bildung zahlreicher grosser Knoten einherging und über fünf Wochen jeder andern Behandlung Trotz bot, wurde durch 23 Tuberkulininjektionen von $1 / 500$ bis $1 \mathrm{mg}$ in sieben Wochen danernd geheilt.

7. Martha Sch., 7 Jahre alt, Rimstedt.

20. IX, 1900. Aufnahme in die Klinik. Familienanamnese belanglos. Ekzem an Nase und Mundwinkeln, seit einer Reihe von Wochen heftige Entzündung des linken Auges. Langen normal (medizinische Klinik).

Status: Rechtes Auge gesund.

Linkes Auge: Starke ciliare Injektion, Cornea leicht ranchig, Vorder" kammer abgeflacht, Kammerwasser getrübt, in der Iris medial vier grosse vaskularisierte Tuberkelknoten, welche die Cornea fast berühren, sieben kleinere miliare Knötchen liegen rund um den erheblich verdickten Pupillarrand, der total mit der vordern Kapsel verwachsen ist. Einige weitere Knötchen in den mittlem Teilen der Iris. Pupillargebiet durch ein graues Exsudat verschlossen. Lichtschein unsicher, keine Projektion.

Obgleich in diesem Fall die Wiedererlangung von Sehvermögen von vornherein ausgeschlossen war, entschloss ich mich doch zur Tuberkulinbehandlung, um die Enueleation wenn möglich za vermeiden, die ja bei kleinen Kindern immer nachteilige Folgen für die Entwicklung der Gesichtsknochen hat.

Vom 23. IX. 1900 bis 5. II. 1901 wurden 56 T. R.-Injektionen von $1 / 500$ bis $3 / 50 \mathrm{mg}$ gemacht, meist Tag um Tag, anfangs um $1 / 500$, dann um $1 / 50 \mathrm{mg}$ steigend. Temperatursteigernng auf 38 bis $39^{\circ} \mathrm{kam}$ nur zweimal ror und ging schnell zurück, so dass die Injektionen nur wenige Tage ausgesetzt wurden.

Drei Wochen nach Beginn der Injektionen zeigten die vier grossen Knoten bereits eine deutliche Verkleinerung, zwischen ihnen traten narbige

v. Graefe's Archiv für Ophthalmologie. LIX, 1. 
Streifen im Irisgewebe auf, ein Teil der kleinen Knötehen war versehwun den, am Orte ihres frühern sitzes die Iris deutlich atrophiert. Die Vaskularisation der Iris ersehien geringer, doch setzten sich einzelne neugebildete Gefässe bis in das Pupillarexsudat fort.

Nach weitern $2 \frac{1}{2}$ Wochen kam es vorübergehend noeh einmal $z u$ vermehrter eiliarer Injektion, leichter Trübung des Kammerwassers, stärkerer" Vaskularisation der Iris und etwas Druekempfindlichkeit des Corpus eiliare innen oben unter leichter Vorbuckelung der Sklera an der schmerzhaften Stelle, doch gingen die Entzïndungserscheinungen im Lanfe von 10 Tagen zurück und der sklerale Buckel verschwand wieder.

Unter fortgesetzten Tuberkulininjektionen bildeten sich nummehr die Knoten in der Iris langsam aber kontinuierlich zurück, die Vaskularisation nahm mehr wnd mehr ab, die Atrophie des Irisgewebes breitete sich immer weiter aus und am 8. Ir. 1901 wurde schliesslich folgender Befund festgestellt: Auge völlig reizlos, Cornea und Kammernasser klar, vordere Kammer infolge Vorwölbung der Iris stark abgeflacht, Pupille eng, totale hintere Synechie, dickes organisiertes Pupillarexsudat. Iris überall wo die Knoten sassen grau verfarbt, atrophiseh; dazwisehen einzelne Stellen normalen Irisgewebes. Alle Knoten verschwunden; Lichtsehein vorhanden, Projektion fehlt. Patientin wird geheilt entlassen.

Wegen meiner Übersiedelung ron. Halle nach Göttingen habe jch sie nicht mehr wiedergesehen. Auf briefliche Anfrage erhielt ich am 23.I. 1904 vom Vater der kleinen Patientin die Auskunft, dass das Auge später in der Klinik in Halle enucleiart sei. Herr Kollege Sohmidt-Rimpler hatte die Freundlichkeit, mir über den Grund der Enucleation folgendes zu schreiben: „Martha Sel. kam mit leicht phthisischem Auge $\left(-T_{2}\right)$, Iris atrophiseh, aber noch zwei periphere Knötchen und Reizerscheinungen. Da die Anamnese ergab, dass das Kind wegen Iristuberkulose behandelt war, wurde der Bulbus enucleiert." Aus diesen Worten glaube ich schliessen zu dürfen, dass weniger der derzeitige Zastand des Auges, der doch nichts bedrolliches an sich hatte, als die Rücksicht anf die fruhere Tuberkulose für die Ausfuhrung der Enucleation massgebend gewesen ist. - Über den anatomischen Befund vexdanke ich Herm Dr. Ackermann in Halle, der den Bulbus untersucht hat, folgende Mitteilung: "Der Bulbus ist in den äussern Dimen sionen erhalten. Die Cornea zellig infiltriert, ron Gefässen durehsetzt. Vorderkammer flaeh, Iris mässig atrophisch, durchsetzt you einer Anzahl Knötchen, die aus einkernigen Lymphoeyten bestehen und weder Verkäsung, noch Riesenzellen, noeh Tuberkelbacillen enthalten, dagegen von Gefässen durchzogen sind. Sie ist durch organisierte Exsudatsehwarten vielfach verwachsen mit dem Rest der Linse, von der die Kapsel und ein starker vorderer Kapselstar erhalten ist, wäbrend die Cortienlsubstanz gröstenteils resorbiert ist. Im Glaskörper" ein dichtes, weisses, kömiges Exsudat. Die Chorioidea frei von frischen Entzindungserscheinungen, bindegewebig atrophiert, die Retina und Papille mässig entzindet, von letzter zieht ein organisierter Strang in das Glaskorperexsudat hinein."

Epikrise: Bei einem 7jährigen, an schwerster Iristuberkulose erkrankten Kinde ist es im Gegensatz zu der notorischen Erfolg- 
losigkeit jeder andern Behandlungsmethode gelungen, lediglich durch 56 T. R.-Injektionen alle Tuberkelknoten zur Rückbildung zu bringen, die Entzündung zu beseitigen und das Auge zu erhalten. Dass die später nochmals aufgetretenen Reizerscheinungen, welche zur Enucleation des Auges Veranlassung gaben, nicht durch ein Rezidiv der Tuberkulose bedingt waren, ist durch die anatomische Untersuchung festgestellt. Der Fall bietet insofern ein besonderes Interesse, als er die Heilbarkeit schwerer Tuberkulose des Auges durch Tuberkulininjektionen nicht nur klinisch, sondern auch anatomisch beweist.

8. Heinrich Sch., 26 Jahre alt, Kl. Battloff.

17. IX. 1901 Aufnahme. Patient hat im Jahre 1896 bereits einmal eine Entzindung des rechten Anges gehabt und will seitdem darauf etwas schlechter sehen. Vor sechs Wochen entzündete es sich abermals, seit vier Tagen verschlimmerte sich der Zustand sehr erheblich. Lues negiert, objektive Zeichen derselben fehlen. Herz und Lungen normal (medizinische Poliklinik).

Status. Rechtes Auge: Lebhafte ciliare Injektion, Tränen, Lichtscheu. Unten und aussen in der Cornea einige tiefe parenchymatöse Trübungen, Kammerwasser leicht getrübt, Iris hyperämisch, zahlreiche Synechien, dünnes $\mathrm{Pu}$ pillarexsudat, Hintergrund normal, $\mathrm{E}, \mathrm{S}<0,5$. Linkes Auge normal, $\mathrm{S}=1$.

Unter dem Gebrauch von Atropin und feuchtwarmen Verbänden lösten sich die Synechien bis auf zwei breite aussen und unten, aber die heftigen Reizerscheinungen hielten an und in der Cornea traten zahlreiche, in den tiefsten Schichten sitzende Infiltrate auf, zwischen denen das Gewebe sich rauchig trübte. Am 30. IX. wurden unten in der Trisperipherie zwei gelbliche vaskularisierte Knötchen sichtbar und zahlreiche feine Gefässe zogen in den tiessten Schichten der Cornea zu den Infiltraten hin. Im Laufe der nächsten 14 Tage nahmen letztere an Zahl noch erheblich $\mathrm{zu}$, so dass am 12. X. mit der binokularen Lupe ungefăr 20 gezählt wurden, die alle dicht vor der Descemetis sassen. Zugleich entwickelten sich unten im Kammerfalz noch zwei nene vaskularisierte Knötchen, es wurde daher am 15. X. mit T. R.-Injektionen begonnen. Da es mir bei der Schwere der Hornhauterkrankung erwünscht war, mögliehst bald eine Wirkung zu erzielen, wählte ich die erste Dosis höher als sonst: ${ }^{5 / 500} \mathrm{mg}$ und steigerte dieselbe schneller, so dass bei der siebenten Injektion bereits $5 / 50 \mathrm{mg}$ zur Verwendung kamen. Patient reagierte auf diese mit einer Temperatur von $40,2^{\circ}$ und Kopfschmerzen, daher wurden die Injektionen drei Tage ausgesetzt und dann mit $2 / 50 \mathrm{mg}$ wieder aufgenommen. Eine langsame Erhöhung der Dosis um 1/50 $\mathrm{mg}$ vertrug der Kranke nunmehr gut bis zur Stärke von ${ }^{8}{ }_{50} \mathrm{mg}$, auf die wieder eine Temperatursteigerung bis $40^{\circ}$ eintrat. Unter diesen Umständen hielt ich es für zweckmässig, nochmals auf die Anfangsdosis herunterzugehen und langsamer zu steigen. Nun blieb jede allgemeine Reaktion aus. Vom 15. X. 1901 bis 10. I. 1902 wurden 36 Injektionen gemacht.

Nach der 12. Injektion zeigte sich eine deutliche Abnahme der Entzündungserscheinungen am Auge, zwei der Knötchen in der Iris waren ver- 
schwunden, die tiefen Infiltrate in der Cornea weniger zablreich, die Trübungen etwas liehter. Vier Wochen später verschlimmerte sich der Zustand vorübergehend wieder, indem Mitte Dezember noch einmal frische Infiltrate auftraten und die Iritis sich steigerte, während zugleich von allen Seiten tiefliegende, neugebildete Gefässe 2 bis $3 \mathrm{~mm}$ weit in die Cornea hineinzogen.

Der Visus sank dabei bis auf Fingerzählen in $2 \mathrm{~m}$. Die definitive Besserung begann mit dem Verschwinden der letzten Knötchen in der Iris Anfang Februar 1902. Die Hornhauttrübungen lichteten sich langsam, aber konstant; die Tritis kam zum Ablanf, das Pupillarexsudat zur Resorption, die Synechien lösten sich zum grossen Teil, Am 18. III. 1902 war $\mathrm{S}=0,1$, am 30. IV. $\mathrm{S}=0,5$, am 23. V. $\mathrm{S}=0,7$. Bei der letzten Vorstellung am 22. VII. 1902 war das Auge reizlos; im Parenchym der Cornea eine Anzahl ganz lichter Maculae und vereinzelte feinste Gefässe, Iriszeichnung seharf, von den Knötchen keine Spur sichtbar, einige hintere Synechien, Pupille aber gut reagierend, in ihr noch einige feinste Exsudatreste, Hintergrund normal, $\mathrm{S}=0,8$.

Epikrise: In dem vorstehenden Fall traten die entzündlichen Veränderungen der Cornea gegenïber denen der Iris so stark in den Vordergrund, dass es manchem vielleicht zweifelhaft erscheinen wird, ob es sich hier um eine tuberkulöse Erkrankung gehandelt hat. Ich glaube eine solche annehmen zu dürfen, weil einerseits alle Anhaltspunkte für Lues fehlen, anderseits die charalteristische Form der Homhautaffeltion, das gleichzeitige Auftreten von Knötchen in der Iris und die lebhafte Reaktion des Patienten auf verhältnismässig niedrige Dosen ron Tuberkulin für Tuberkulose sprechen. Der Versuch, die Behandlung mit grössern Anfangsdosen zu beginnen, um schneller eine Wirkung auf den Krankheitsprozess zu erzielen, hat sich auch in diesem Falle als verfehlt erwiesen, weil die starke Allgemeinrealtion wiederholt ein Aussetzen derselben notwendig machte. Aber auch die Entzindung des Auges ist vielleicht durch die schneller als sonst steigenden Dosen vorübergehend ungünstig beeinflusst worden. Sie ging erst nach dem regelmässigen Gebranch viel kleinerer Dosen definitiv zurück. Besonders bemerkenswert bei der Schwere und Hartnäckigkeit der Erkrankung erscheint mir die durch die Tuberkulinbehandlung erreichte Sehschärfe.

9. W. v. G., 19 Jahre, Cassel.

29. XII. 1902. Patient leidet schon über ein Jahr an einer Entzündung des rechten Auges, wegen deren er von verschiedenen Augenärzten ohne Erfolg behandelt ist. Drüsen vor und hinter dem rechten Obr stark geschwollen, ebenso eine submaxillare Drüse. Kein Zeichen von Lues vorhanden, Lungen und Herz normal. 
Status. Rechtes Auge: Lebhafte eliare und conjunctivale Injektion; in der Cornea, besonders zentral, eine grosse Zahl feinster, punktförmiger Trübungen in den mittlem und hintern Schichten; eine intensiv weissgraue, etwas grössere Trübung in der Höhe des untern Pupillarrandes liegt dicht vor der Descemetis, ihr sehr nahe eine zweite; zn beiden ziehen tiefliegende Gefässe vom Hornhautrande. Der ganze untere Umfang der Iris ist fast bis zum Pupillarrand hin von einer gelblichen, aus einzolnen Knoten konfluierten, an der Oberfläche vaskularisierten Geschwulst eingenommen, die fast die Hinterfläche der Cornea berührt. In der inneren Hälfte der Iris liegen zwei Gruppen von Knötchen, deren untere aus vier, deren obere aus sieben aneinanderstossenden Knötchen besteht. Teilweise sind sie ron einem feinen Gefässnetz übersponnen. Zwischen diesen beiden Knötchengruppen schiebt sich eine dritte, anscheinend ganz frische, im Ciliarteil der Iris gelegene aus dem Kammerwinkel hervor. Eine weitere nimmt oben die ganze Breite der Iris ein. Oben aussen und anssen liegen vier vaskularisierte Knötchen im Kammerfalz; die ganze Iris ist von zahlreichen, erweiterten Gefässen durchzogen, die Pupille etwas erweitert, aber nnregelmässig, überall der Kapsel adhärent, durch ein grauliches Exsudat verschlossen. S Finger dicht vor dem Auge, Projektion normal.

Linkes A uge gesund, $\mathrm{E}, \mathrm{S}=1$.

30. XII. 1902. Beginn der Tuberkulinbehandlung mit 1/500 mg, Tag: um Tag um ${ }^{1 / 500} \mathrm{mg}$ steigend; von ${ }^{1 / 50} \mathrm{mg}$ ab Erhöhung der Dosis um $1 / 50$ mg. Da bei ${ }^{8} / 50$ resp. ${ }^{9} / 50$ mg. Temperatursteigerung bis $38^{\circ}$ eintrat und Patient über Kopfschmerz und allgemeines Unbehagen klagte, wurde einige Wochen hindurch an der Dosis von $5 / 50 \mathrm{mg}$ festgehalten und dann langsam bis anf $1 / 5 \mathrm{mg}$ gestiegen. Im ganzen kamen die Injektionen - abgeseben von einer dreiwöchentlichen Pause im März - bis Mitte September 1903 zur Anwendung; eine nennenswerte Störung des Allgemeinbefindens machte sich niemals bemerkbar, dagegen stieg die Temperatur nach den Injektionen öfters bis auf $38^{\circ}$, um immer nach wenigen Stunden zur Norm zurückzukehren.

Bereits Mitte Januar 1903 begannen die Hornhauttrübungen etwas lichter zu werden, die grossen Gesehwulstknoten in der Iris erschienen kleiner und weniger stark vaskularisiert, einzelne der kleinen Knötchen waren nicht mehr sichtbar.

16. II. 1903. Die Knoten in der Iris unten und unten aussen haben sich völlig zurückgebildet, der innen unten ist flacher und weniger vaskularisiert, an Stelle des einen oberen sieht man in der Iris ein gefässloses Narbengewebe, zwei Knoten oben noch sichtbar, aber kleiner.

Im Laufe der nächsten Monate schritt die Rückbildung der Knoten zwar langsam, aber ununterbrochen fort, die Vaskularisation der Tris nahm immer mehr ab. Die Hornhauttrübungen lichteten sich, die Beschläge an der Descemetis verschwanden, das Auge war völlig reizlos.

Am 17. IX. 1903 wurde folgender Befund festgestell:

In der Cornea unterhalb der Mitte eine lichte Trübung, in der man selbst mit der Zeissschen Lupe kein Gefäss mehr sieht. Alle Tuberkelknoten in der Iris verschwunden, an ihrer Stelle im Irisgewebe atrophische, graue, an den Rändern etwas dunkler pigmentierte Flecken. Pupillarrand 
total adhärent, im Pupillargebiet ein dünnes organisiertes Exsudat; Linse dicht unter der Kapsel leicht getrübt, Hintergrund nicht sichtbar, $\mathrm{S}=0,1$, Projektion normal.

Epikrise: Patient, an schwerster Iristuberkulose leidend, die länger als ein Jahr trotz andauernder sachverständiger Behandlung fortgeschritten war und das Auge mit völiger Zerstörung bedrohte, wurde durch 69 Tuberkulininjektionen im Laufe von acht Monaten völlig geheilt. Dieselben übten - abgesehen ron geringen, schnell rorubergehenden Temperatursteigerungen, die zuweilen mit leichten Kopfschmerzen verbunden waren - nicht den geringsten nachteiligen Einfluss auf das Allgemeinbefinden aus. Seit $1 / 2$ Jahr ist das Auge ron Eutzindung frei geblieben. Eine Besserung des Sehvermögens durch eine spätere Iridektomie lässt sich sicher erwarten.

10. Fräulein E. S., 18 Jahre alt, Nordhausen.

Patientin stammt aus einer tuberkulös belasteten Familie. Eine Schwester ist an Phthisis gestorben. Sie selbst leidet seit der Kindheit an Drissenschwellungen, Rhinitis und Laryngitis chronica. Anfang April 1903 entzündete sich das linke Auge und das Sehvermögen sank sehr erheblich. Am 15. V. 1903 trat Patientin in meine Behandlung.

Status: Mässige ciliare Injektion, die unteren $Z$ weidrittel der Comea an der Hinterfläche mit massenhaften grössern und kleinern Präzipitaten bedeckt, vor ihnen in den tiefsten Schichten der Cornea zahlreiche rundliche Trübungen, Kammerwasser rauchig, Iris verfärbt, etwas hyperämisch. Pupille dureh Atropin erweitert, einzelne periphere Synechen. Glaskörper diffus getrübt, Hintergrand verschleiert, pathol. Veränderungen nicht sichtbar. $\mathrm{M}=2,5 \mathrm{D}, \mathrm{S}=0,2$.

Rechtes Ange normal, $\mathrm{H}=2,5, \mathrm{~S}=1$. An Herz und Lungen nichts Krankhaftes nachweisbar.

Durch wiederholte Punktionen der" vordern Kammer, Atropin, Kataplasmen und subconjunctivale Kochsalzinjektionen gelang es zwar vorübergehend, die Zahl der Präzipitate erheblich zu verringern und den Glaskörper etwas aufzuhellen, aber die Besserung war nie von Dauer. Patientin ging daber Anfang Juli zII einer längern Badekur nach Nauheim. Am 28. IX. 1903 stellte sie sich wieder vor. Ihr allgemeines Aussehen war besser, das Auge äusserlich reizlos, aber an der ganzen untern Hälfte der Descemetis lagen dicke Präzipitate, besonders massig an der Innenfläche der Punktionsnarbe, die Hornhaut davor war stärker getrübt, Flnorescein rief eine tiefliegende Grünfärbung hervor. Im Glaskörper sah man ein vom innern obern Teil des Corpus ciliare ausgehendes graues Exsudat dicht hinter der Linse, ausserdem eine Anzahl beweglicher Flocken. Hintergrund kaum noch erkennbar, S Finger in $3 \mathrm{~m}$.

Bei dieser wesentlichen Verschlimmerung des Zustandes wurde am 30. XX. 1900 mit der Tuberkulinbehandlung begonnen. Anfangsdosis 1/500, mg, Tag um Tag um $1 / 500 \mathrm{mg}$ steigend, von $1 / 50 \mathrm{mg}$ ab um $1 / 50-2 / 50 \mathrm{mg}$. 
Nach Irjektion von $36 / 50 \mathrm{mg}$ einer frisch bezogenen Tuberkulinlösung am 21. XII. 1903 stieg die Temperatur, die bis dabin normal geblieben war, plötzlich auf $40^{\circ}$ unter gleichzeitigem Kopfschmerz und dem Gefühl allgemeiner Abgeschlagenheit, kehrte aber in wenigen Stunden zur Norm zurück. Da der gleiche Vorgang sich nach den beiden nächsten Injektionen wiederholte, ging ich mit der Dosis nochmals auf $10 / 50 \mathrm{mg}$ herunter, um dieselbe dann wieder jedesmal um $4 / 50 \mathrm{mg}$ zu erhöhen. Über ${ }^{40 / 50} \mathrm{mg}$ bin ich nicht hinausgegangen, da fluchtige Temperatursteigerungen auf $38^{\circ}$ schon nach niedrigeren Dosen an einzelnen Tagen vorkamen. Im ganzen wurden 68 Tuberkulininjektionen bis zum 29. II. 1904 gemacht.

Während der Behandlung trat am 14. X. 1903 ein kleines raskularisiertes Knötchen innen unten in der Iris auf, das im Laufe von sechs Tagen wieder verschwand. Ohne Zunahme der Injektion des Bulbus wurde am 27. X. 1903 die Sklera am innern obern Comealrand ein wenig ektatisch unter gleichzeitiger geringer Druckempfindlichkeit des Corpus ciliare an dieser Stelle und die Präzipitate an der Cornea erschienen noch zahlreicher, während Exsudation und Trübung im Glaskörper noch unverändert waren. Die deutliche Aufhellung desselben begann am 5. XI. 1903 und sehritt dann ununterbrochen fort, ebenso versebwanden allmählich die Präzipitate und Trübungen der Hornhant mehr und mehr and die Ektasie der Sklera ging zurück.

Bis zum 10. XII. 1903 war das grosse Glaskörperexsudat grossenteils resorbiert, an seiner Stelle nur noch zwei kleine bewegliche Flocken vorhanden, der übrige Glaskörper soweit klar, dass man den Hintergrund wieder ziemlich deutlich sah. $S=0,2$. Unten aussen in der Iris zeigte sich abermals ein kleines vaskularisiertes Knötchen, das im Laufe von vier Tagen wieder verschwand.

Unter weiteren, Tag um Tag ausgefuihrten Tuberkulininjektionen hellte sich der Glaskörper wieder völlig auf, so dass man ein deutliches Bild des Augenhintergrundes erhielt, der keine pathologischen Veränderungen zeigte. Die Präzipitate an der Descemetis wurden verbältnismässig langsam resorbiert, verschwanden aber doch in den beiden nächsten Monaten völlig. Einzelne derselben hinterliessen kleine grane Trübungen in dem Fndothel der Cornea, die ror dem untern Teil der Pupille liegen und daher das Sehvermögen etwas beeinträchtigen. Immerhin hat sich $\mathrm{S}$ bis auf 0,8 von Fingerzählen in $3 \mathrm{~m}$ gehoben und Patientin konnte am 9. III. 1904 geheilt entlassen werden.

Epikrise: Dieser Fall unterscheidet sich von den meisten vorher mitgeteilten durch eine andere Lokalisation des tuberkulösen Prozesses. Das mächtige Exsudat im Glaskörper dicht hinter der Linse, sowie die massenhaften Präzipitate an der Descemetis wiesen in Verbindung mit der vorübergehenden Ektasie der Sklera am innern obern Cornealrande auf eine primäre Erkrankung des Ciliarkörpers hin, während die Tris nur in geringem Grade sekundär in Mitleidenschaft gezogen war. So kam es nur zur Bildung einzelner Synechien, während das Pupillargebiet völlig frei blieb. Bemerkens- 
wert erscheint mir der Einfluss des Tuberkulins nicht nur auf den im Corpus ciliare lokalisierten tuberkulösen Herd, sondern auch auf das durch ihn hervorgerufene dicke Glaskörperexsudat, welches zu völliger Resorption gelangte und zwar schneller als die Präzipitate an der hintern Hornhautwand.

Den mitgeteilten zeln Fällen von Tuberkulose der Cornea und des Ũvealtractus lasse ich zunächst den Bericht iiber drei Fälle von Tuberkulose der Conjunctiva und einen von Tuberkulose der Sklera folgen, um dann die Resultate der Tuberkulinbehandlung kuxz zusammenzufassen.

Anna Z., 11 Jahre alt, aus Wünschendorf.

Aufnahme 3. III. 1900. Hereditäre Belastung nicht vorhanden. Seit dem Herbst $v$. J. besteht eine Entzündung des rechten Anges, die bisher. vergeblicb ärztlich behandelt wurde.

Status: Lider rechts gerötet und verdickt, besonders in der Gegend der Ränder. Nach Ektropionierung sieht man in der Conjunctiva des untern in der temporalen Hälfte eine Gruppe follikulärer Wucherungen, von der Übergangsfalte bis zum intermarginalen Teil reichend, dazwisehen eine flache papilläre Exerescenz von Erbsengrösse. In der nasalen Hältte der Conjunctiva des obern Lides eine linsengrosse papilläre Wucherung umgeben von follikulären Gebilden, in ihrer Nähe noch mehrere einzeln stehende Zapfen. Cornea und Conjunetiva bulbi normal, ebenso Hintergrund, $\mathrm{S}=1$.

Linkes Auge gesund, $\mathrm{S}=1$.

Am 5. III. wurden von den papillären Wucherungen kleine Partikel abgetragen und in die vordere Kammer eines Kaninchens implantiert, am 31. III. kam bei diesem typische Iristuberkulose zum Ausbruch.

Da eine Behandlung mit Jodoform und Verband erfolglos blieb, wurde am 4. IV. mit Tuberkulininjektionen begonnen. Anfangsdosis $1 / 500 \mathrm{mg}$, Tag um Tag Steigerung um $1 / 500 \mathrm{mg}$. Nach der zweiten und dritten Injektion Rötung und Infiltration an den Einstichstellen mit Temperatursteigerung auf 38,1 resp. 38,5 , die schnell wieder versehwindet.

13. IV. Blässe und Koptschmerzen. Unterhalb des Tränensackes nach der Nase hin eine pfenniggrosse, umsehriebene Rötung und Schwellung der Haut, welche auf Druck schmerzhaft ist. Dieselbe verbreitete sich bei ungestörtem Allgemeinbefinden in den nächsten drei Tagen über den Nasenriueken bis auf die linke Wange, ging aber bis zum 20. IV. spontan zurück, so dass am 25. IV. die Injektionen wieder aufgenommen und nunmehr regelmässig fortgesetzt werden kounten.

3. V. Die Wucherungen auf der Conjunctiva beider Lider beginnen kleiner und flacher $\mathrm{zn}$ werden, die vereinzelt stehenden Excrescenzen sind bis auf drei zurückgegangen, Injektion und Schwellung der Conjunctiva nehmen $a b$. 
14. V. Injektion von ${ }^{1 / 50} \mathrm{mg} \mathrm{T}, \mathrm{R}$; von nun ab Steigerung der Dosis um $1 / 50 \mathrm{mg}$ bis za ${ }^{6 / 50} \mathrm{mg}$ am 25. V., worauf die Temperatur auf $38,6^{0}$ stieg; daher Schluss der Behandlung nach 18 Injektionen am 1. VI. 1900 .

Im Laufe des Mai hatten sich die Wucherungen immer mehr abgeflacht, an ihrer Stelle sah man eine glatte Narbe in der Conjunctiva, die Schwellung der Lider war zurückgegangen, ihre Stellung normal. Dagegen zeigte die Bindehaut bei der Entlassung noch rermehrte Injektion.

Am 23. II. 1901 kam Patientin nochmals zur Aufnahme mit einem leichten Rezidiv in der Conjunetiva des untern Lides. An der innern Lidkante, im lateralen Drittel, befindet sich ein ungefähr $3 \mathrm{~mm}$ langes, ganz flaches Uleus mit rötliehem Grund und grau gefärbten Rändern, das auf den freien Lidrand übergreift.

Nach drei weitern Tuberkulininjektionen stellte sich das Epithel schnell her und das Geschwür kam im Laufe von 8 Tagen zur Heilung. Die Patientin hat sich nicht wieder vorgestellt, aber auf briefliche Anfrage sehreibt mir am 26. I. 1904 der Vater, dass das Auge vollständig gesund geblieben sei.

Elise D., 7 Jahre alt, Hettstedt, war bis zum 1. IV. 1900 gesund. Damals erkrankte ohne nachweisbare Veranlassung ihr rechtes Auge. Da jede Behandlung fruchtlos blieb, wurde sie am 5. XI. 1900 in die Klinik aufgenommen. Lungen gesund, hereditäre Belastung nicht vorhanden. Linkes Auge normal.

Rechtes Auge: Mässige Ptosis. Haut des obern Lides, besonders in der medialen Hälfte, etwas gerötet und geschwellt, Conjunctiva beider Lider injiziert, ziemlieh starke Sekretion. In der medialen Hälte der Conjunctiva tars. sup. ein ungefähr fünfpfennigstückgrosses flaches Ulcus mit unebenem Grund und dünnem weisslichgranen Belag. Seine Ränder sind nicht unterminiert, auch nicht stärker infiltriert.

Das Uleus reicht über die innere Lidkante bis auf den intermarginalen Teil, nach oben zu fast bis zur Übergangsfalte. Augapfel selbst normal.

7. XI. Impfung eines Kaninchens, 4. XII. Ausbruch typischer Iristuberkulose.

Auch in diesem Fall wurde zunächst die Jodoformbehandlung ohne Erfolg versucht, sodann aber von 20. XI. ab zu Tuberkulininjektionen übergegangen. Die Dosierung war dieselbe, wie in dem vorigen Fall. Trotzdem traten nach den sieben ersten Injektionen regelmässig am zweiten Tage "Temperatursteigerungen auf, die im Laufe von zwei Stunden ihr Maximum mit $38,6^{\circ}$ bis 39,1 erreichten, um dann fast ebenso schnell wieder zu verschwinden.

Eine Störung des Algemeinbefindens trat dabei nicht ein. Von der dritten Woche der Behandlung an erhob sich die Temperatur nur noch ausnahmsweise über $38^{\circ}$, obgleich die Dosen allmählich bis auf $20 / 50 \mathrm{mg}$ gesteigert wurden. Im ganzen wurden 35 Injektionen gemacht, die letzte am 25. II. 1901.

Mitte Dezember erschien die Begrenzung des Geschwürs schon weniger 
A. v. Hippel.

scharf, die papillikren Wucherungen hatten sich verkleinert, der Grund des Geschwürs zeigte keinen Belag mehr, sondem eine reichliche Gefässneubildung, das Epithel der Conjunctiva begann sich über die Ränder herïberzuschieben. Bis Ende Dezember war die Heilung so weit vorgeschritten, dass der Grund des Geschwïrs in einem Nireau mit der angrenzenden Conjunctiva lag und sich völig geglättet hatte. Von den Ründern her trat nun im Laufe der nächsten Wochen die Vernarbung ein. Am 26. I. war der ganze fruhere Geschwirsgrund in ein graues, gefässarmes Narbengewebe umgewandelt, dessen Oberfäche spiegelte, Nur in der Nähe der innern Lidkante fand sich noch ein stecknadelkopfgrosser flacher Substanzveriust. Bis zum 15. II. hatte sich auch dieser gefüllt und Patientin wurde am 28. II. 1901 obne Stellungsanomalie des Lides geheilt entlassen. Anf briefliche Antrage berichtet am 29. I. 1904 der Vater, dass das Kind ungefahr zwei Jahre nach Abschluss der Behandlung gestorben sei. Todesursache unbekannt.

Cari A., 12 Jabre alt, Eschershausen.

Eltern gesund. Patient hat früher viel an Ausschlägen im Gesicht gelitten, aber gesunde Augen gehalut. Im Dezember 1900 entzindete sich das linke Auge. Da eine balbjährliche ärzliche Behandiung keinen Erfolg hatte, kam Patient am 10. VI. 1901 zur Aufnahme in die Klinik.

Status: Innere Organe und rechtes Auge gesund.

Linkes Auge: Oberlid etwas gerötet und so geschwollen, dass es kaum spontan gehoben werden kann. Unterlid äusserliclı normal. Ziemlich reichliche schleimig-eitrige Sekretion. Die innern zwei Drittel der Conjunctiva des obern Lides werden von dicht gedrängt liegenden papillaren Wucherungen eingenommen, die von tiefen Einschnitten durchsetzt sind. Dieselben reichen vom freien Lidrand bis in die Übergangsfalte. Inmitten der Wucherungen findet sich ein Geschwür mit schmierig belegtem Grunde. Conjunctiva bulbi etwas injiziert, in der obern Hälfte der Connea eine Anzahl oberflächlicher Trübungen, zu denen einzelne Gefässe ziehen; untere Hälfte ziemlich klar. Auge im äbrigen gesund, $\mathrm{E}, \mathrm{S}<0,2$.

Linke präaurikulare nnd submaxillare Drüsen stark gesehwollen, hart, aber nicht schmerzhaft, Haut daruber normal. Impfung eines Kaninehens mit positivem Resultat. Patient wurde rom 11. VI. bis 28. XI. mit Tuber kulininjektionen behandelt. Da dieselben in den ersten zwei Monaten regelmässig sehnell voribergehende Temperatursteigerungen von 38,1 bis $39^{\circ}$ hervoriefen, so stieg ich mit den Dosen langsamer als sonst und machte grössere Intervalle zwischen den einzelnen Injektionen. Im ganzen kamen davon 69 zur Verwendung, die Maximaldosis betrug $20 / 50 \mathrm{mg}$. Wiederholt bildeten sich an der Einstichstelle trotz strenger Asepsis kleine Anschwellungen, die zum Teil abscedierten, meistens aber nach drei bis wier Tagen wieder zurückgingen.

Nach Ablauf des exsten Monats der Behandlung war Schwellung und Rötung des obern Lides wesentlich geringer, von der Lidkante her zogen Narbenzüge gegen das Geschwür hỉ, die papillären Wucherungen erschienen flacher, der Grund des Vleus zeigte keinen Belag mehr.

15. VIIr. Weitere Abflachung der Wucherungen, vom obern Geschwïrsrande schreitet die Vernarbung fort. 
13. IX. Da die Rückbildung der papillären Wucherungen sehr langsam erfolgt, werden sie mit Flachbrenner leicht kauterisiert.

Dies Verfahren wurde im September und Oktober noch dreimal wiederholt. Es hatte zwar eine Abflachung und Glättung des Gescliwürsgrundes zur Folge, aber es kam dabei zu keiner Regeneration des Epithels und die Tuberkulininjektionen mussten daher fortgesetzt werden. Erst im November machte die Fernarbung schnellere Fortschritte.

15. XI. Das Uleus ist bis auf eine kleine Stelle von Hirsekorngrösse epithelisiert; seine Oberfläche liegt nur ganz wenig unter dem Niveau der angrenzenden normalen Conjunctiva. Schwellnng des obern Lides fast verschwunden.

1. XII. An Stelle des Ulcus eine ganz flache narbige Delle in der Conjunctiva, die sich gegen die normale Schleimhaut scharf abgrenzt. Sie ist abberall von Epithel bedeckt. Patient wird geheilt entlassen. Stellung des Lides normal.

1. II. 1902. Ange reizlos, Geschwïr geheilt.

14. II. 1904. Patient berichtet brieflich, dass sein Auge gesund geblieben.

Epikrise: In drei Fällen schwerer Tuberkulose der Conjunctiva, welche jeder andern Behandlung ein halbes Jahr lang Trotz geboten hatte, wurde durch Tuberkulininjektionen dauernde Heilung ohne jede Stellungsanomalie der Lider erreicht. Wenn in dem letzten Fall der Heilungsprozess langsame Fortschritte machte, so erllärt sich dies ungozwungen durch die geringere Steigerung der einzelnen Tuberkulindosen und die längern Pausen zwischen den Injektionen, welche durch die Temperatursteigerungen bedingt waren. Dass die Anwendung des Galvanokauters allein das tuberkulöse Geschwür nicht zür Heilung zu bringen vermochte, geht aus der Krankengeschichte zur Genüge hervor. Als bemerkenswerten, Erfolg der Tuberkulintherapie möchte ich das Fehlen jeder tiefgreifenden Narbenbildung in der Conjunctiva hervorheben, die bei andern Behandlungsmethoden nicht auszubleiben und fehlerhafte Lidstellungen zur Folge zu haben pflegt.

Zum Schluss lasse ich noch einen kurzen Bericht über einen Fall von Tuberkulose der Sklera und Cornea folgen.

Friedrich E., 19 Jahre alt, ans Ellrich, hat als Kind an Tuberkulose der Tibiae und wiederholten Augenentzündungen gelitten. Vor $4{ }_{1}^{1} / 2$ Jahren erkrankte er an einer Otitis, wegen deren ihm der Processus mastoidens. aufgemeisselt wurde. Seit ungefähr zehn Tagen besteht eine Entzündung des linken Auges, wegen deren er am 22. VII. 1903 in die Klinik aufgenommen wurde.

Status: Rechtes Auge äusserlich reizlos; am Limbus erstrecken sich 
alte parenchymatöse Trübungen rings herum mehr weniger weit in die Cornea hinein, deren Zentrum klar ist. Iris etwas atrophiseh, Pupille unregelmässig, zahlreiche Synechien. Medien klar, Hintergrund normal, H 5 D, $\mathrm{S}=0,6$.

Linkes Auge: Conjunctivale und ciliare Injektion, besonders stark im untern Umfange der Cornea, wo auf der Sklera eine Anzahl über stecknadelkopforosser Knötchen von gelbgraner Farbe sitzen, über denen die Conjunctiva verschieblich ist.

Thre Basis ist von einem feinen Gefässnetz umsponnen, drei reichen bis an den Hornhautrand heran. Im obern äussern Quadranten der Cornea einige tiefliegende Trübungen, zwischen denen sich feine Gefässe verästeln. Kammerwasser klar, Iris etwas verfärbt, zahlreiche alte Synechien, Medien transparent, Hintergrund normal, $\mathrm{H}=1 \mathrm{D}, \mathrm{S}=0,6$.

Vom 23. VII. bis 12. VIII. elf Tuberkulininjektionen von $1 / 500$ bis ${ }^{1 / 50} \mathrm{mg}$. Während derselben nahm die Injektion des Auges ab und die Knơtchen bildeten sich zuriek.

21. VIII." Nur noeh minimale pericorneale Injektion, Knötchen in der Sklera kaum noch sichtbar, daher auf Wunsch des Patienten Entlassung.

14. IX. Rezidiv. Linkes Ange wieder lebhaft injiziert, eine ganze Anzahl von Knötchen in der Sklera aussen und innen vom Hornhautrande, einige auch unten aussen in der Cornea selbst. Hnmor aqueus klar, Iris nicht gereizt.

17. X. Wiederanthahme der Tuberkulininjektionen, deren 19 in der Dosis rom $1 / 500$ bis $1 / 5 \mathrm{mg}$ bis zum 23. XI. zur Verwendung kamen.

Abermals ging die Injektion schnell zurück, ebenso versehwanden die episkleralen Knötchen, dagegen konfluierten zwei in den tiefen Schichten der Cornea gelegene unter gleichzeitiger Zunahme der Vaskularisation. Ehe diese zur Ritckbildung gelangten, musste Patient leider anf Verlangen der Krankenkasse abermals rorzeitig entlassen werden.

Ein im März 1904 gemachter Versuch, durch briefliche Anfiage Auskunft über das weitere Verhalten des Auges zn erhaiten, blieb erfolglos, da Patient seinen Wohnort verlassen hatte und nicht aufaufinden war.

Überblicken wir nummehr die durch die Tuberkulinbehandlung in unsern Fiallen erreichten Resultate, so dürfen dieselben wohl besonders im Hinblick auf die Schwere der Erkrankung als höchst erfreuliche bezeichnet werden. In allen, mit Ausnahme des letzten, in dem die Behandlung vorzeitig abgebrochen werden musste, gelangte die Tuberkulose zur Heilung, zweimal (Fall 2 und 7) selbst in solchen Augen, welche vor Beginn der Behandlung bereits ihr Sehvermögen eingebüsst hatten. Eine gute Selsschärfe $(S=0,8)$ wurde dreimal (Fall 5, 8, 10) erreicht, geringere Sehleistungen in den Fällen $9(S=0,1), 1,3,4,6$ (Fingerzählen in 5 bis 1 Meter). Die Herabsetzung des Visus in der letzten Gruppe war in erster Linie durch das Vorhandensein von 
organisierten Pupillarexsudaten verursacht. Durch eine später auszuführende Tridektomie wird sich also voraussichtlich noch eine weitere Besserung erzielen lassen.

Ohne jede Stellungsanomalie der Lider gelangten alle drei Fälle von schwerer Conjunctivaltuberkulose zur Heilung; in dem einzigen Fall von Tuberkulose der Sklera waren bei der Entlassung des Patienten alle Knoten verschwunden.

Von besonderer Wichtigkeit ist natiurlich die Beantwortung der Frage, ob die durch die Tuberkulintherapie herbeigeführten Heilungen vorübergehende oder dauernde waren. Durch briefliche Erkundigungen bei allen frühern Patienten habe ich versucht dieselbe $\mathrm{zn}$ entscheiden und dabei folgendes festgestellt:

Fall 1. Emma H., geheilt seit $9^{1 / 2}$ Jahren.

2. Minna B., Wohnort nicht zu ermitteln.

3. Berthold B., Heilung 2 Jahre selbst beobachtet.

4. Minna H., geheilt seit 7 Jahren.

5. Marie K., Heilung 5 Jahre selbst beobachtet.

6. Hermann A., geheilt seit 4 Jahren.

7. Marta Sch., geheilt 2 Jahre, dann von SchmidtRimpler enucleiert, anatomisch keine Tuberkulose gefunden.

" 8. Heinrich Sch., Heilung $1 / 2$ Jahr beobachtet; jetziger Wohnort nicht zu ermitteln.

9. v. G., geheilt; Beobachtungszeit 6 Monate.

" 10. Fräulein E. S., eben geheilt entlassen.

"11. Anna Z., geheilt seit 3 Jahren.

" 12. Elise D, 2 Jahre geheilt, dann gestorben.

"13. Carl A., geheilt seit 2 Jahren.

"14. Friedrich E., Wohnort nicht zu ermitteln.

Auf Grund der von mir mitgeteilten Thatsachen balte ich den Beweis für erbracht, dass wir in dem Tuberkulin T. R. ein Mittel besitzen, welches - richtig angewandt - selbst schwerste Tuberkulose des Auges mit Erhaltung von Sehvermögen dauend zu heilen vermag. Hieraus erwächst uns meines Erachtens die Pflicht, nicht, wie es bisher vielfach geschah, derartige Augen ohne weiteres zu enucleieren, sondern zunächst die Bekämpfung des Leidens mit den Waffen zu versuchen, die wir $R$. Koch zu verdanken haben. Wir dürfen dies um so unbedenklicher tun, als die Erfahrung gelehrt hat, dass bei der Tuberkulose des Auges die Tuberkulininjektionen in den kleinen Dosen, wie wir sie nur brauchen, niemals nachteilige Folgen 
30 A. v. Hippel, Über den Aiutzen des Tuberkulins bei der Tuberkulose des Auges.

für das Allgemeinbefinden der Patienten haben, während in einer Anzahl ron Fällen nach der Enucleation Tod durch Meningitis eintrat ${ }^{1}$ ).

Ob bei dieser Therapie die Erhaltung aller tuberkulös erkrankter Augen gelingen wird, kann erst die Zukunft entscheiden; das Material des einzelnen reicht bei der Seltenheit des Leidens nicht aus, um diese Frage zu beantworten. Wird aber auch nur ein erheblicher Teil gerettet - und diese Annahme dürfen wir im Hinblick auf meine Resultate als sicher begriindet betrachten -, so haben wir damit einen erfreulichen Fortschritt auf therapeutischem Gebiet zu verzeichnen, der uns allein durch die genialen Arbeiten Robert Kochs ermöglicht worden ist.

Göttingen, März 1904.

1) Rogman, Tuberculose intraoculaire. Dangers de l'énucléation. Annales d'oculist. Tome CXXX. 1903. p. 65. 